Received: 28 November 2017

Accepted: 25 April 2018

Published online: 09 May 2018

\section{Supremum of block entanglement for symmetric Gaussian states}

\author{
Jhih-Yuan Kao \& Chung-Hsien Chou
}

For a system composed of permutationally symmetric Gaussian modes, by identifying the boundary of valid states and making necessary change of variables, the existence and exact value of the supremum of logarithmic negativity (and negativity likewise) between any two blocks can be shown analytically. Involving only the total number of interchangeable modes and the sizes of respective blocks, this result is general and easy to be applied for such a class of states.

Entanglement, as a distinct characteristic of quantum physics from which many interesting properties stem, such as nonlocality, has been shown to be a potential resource for quantum information ${ }^{1-10}$. Among various quantum systems of interest are continuous variable (CV) systems, of which Gaussian states have been a focus of researches, so has the entanglement and measures of information thereof ${ }^{11-22}$. A Gaussian state is a CV state whose Weyl (or Wigner) characteristic function is Gaussian, which results in a Gaussian Winger quasi-probability distribution ${ }^{22}$. A symmetric Gaussian state is one that is invariant under interchange of modes, which has garnered interests over the years ${ }^{23-27}$.

The search for bounds of entanglement have been conducted for different kinds of systems in terms of various measures, e.g. entanglement of formation ${ }^{28}$ for two-mode Gaussian states ${ }^{29,30}$ and geometric measure of entanglement ${ }^{31-33}$ for symmetric qubits ${ }^{34-36}$.

The system of interest in this work is multiple modes in a symmetric Gaussian state, which is invariant under mode exchanges. We will discuss the bipartite entanglement between two blocks of modes, both of which are parts of the aforementioned modes. We're going to first talk about some basic properties of Gaussian states, and then of symmetric Gaussian states, the primary subject of this work. Building upon this basis, we will show how to characterize a symmetric Gaussian state with proper variables so that a simple constraint can be established, essential for ascertaining the supremua (least upper bounds) of entanglement, with respect to negativity and logarithmic negativity ${ }^{37}$. Attributes and implications of these suprema will be discussed thereafter.

\section{Entanglement of Gaussian states}

Covariance matrix, partial transpose and Gaussian states. The covariance matrix of quadrature operators $x_{i}$ and $p_{i}$ is composed of variances and covariances of them:

$$
\sigma_{i j}=\sigma\left(\xi_{i}, \xi_{j}\right),
$$

where $\sigma(A, B)=\langle\{A-\langle A\rangle, B-\langle B\rangle\}\rangle / 2$ denotes the covariance of operators (variables) $A$ and $B$ (variance if $A=B)$ and $\xi=\left(x_{1}, p_{1}, x_{2}, p_{2}, \ldots\right)^{\mathrm{T}}$, the subscript of $x$ and $p$ for the index of parties. For a continuous variable system, because of the commutation relation $\left[x_{i}, p_{j}\right]=i \hbar \delta_{i j}$, the covariance matrix of a valid state should satisfy this condition $^{38}$

$$
\sigma+i \hbar \Omega / 2 \geq 0,
$$

where $\Omega=\operatorname{diag}\left(\left(\begin{array}{cc}0 & 1 \\ -1 & 0\end{array}\right),\left(\begin{array}{cc}0 & 1 \\ -1 & 0\end{array}\right), \ldots\right)$, which is an alternative form of the commutation relation. By Peres criterion $^{39}$, the partial transpose of a separable state must be positive. Note that $\hbar$ is kept throughout this paper, so whichever convention is adopted or whichever system is considered the result can be easily adjusted. The transpose of a CV state corresponds to a reversal of momentum, which results in Peres-Horodecki-Simon criterion ${ }^{39-41}$ for separability

$$
\widetilde{\sigma}+i \hbar \Omega / 2 \geq 0
$$


where the tilde indicates the reversal of momentum of the transposed party, hence partially transposed. This in general is a necessary condition for separability, but not sufficient. Assuming the first moments are zero, achievable by local unitary operations, a Gaussian state can be totally characterized by its covariance matrix ${ }^{22,41}$ :

$$
W(\xi)=\mathcal{N} \exp \left(-\frac{1}{2} \xi^{\mathrm{T}} \sigma^{-1} \xi\right)
$$

where $W$ is the Wigner distribution and the normalization factor $\mathcal{N}$ depends on $\sigma$ and the number of modes. Thus, the covariance matrix can be thought of as a representation of a Gaussian state, and sometimes we'll just call the covariance matrix $\sigma$ the "state".

Logarithmic negativity. Since logarithmic negativity ${ }^{37}$ measures "how negative" the state is after partial transpose, defined as (a particular base isn't assigned for flexibility)

$$
E_{\mathcal{L}} \equiv \log \left\|\rho^{\mathrm{T}_{A}}\right\|_{1},
$$

where $E_{\mathcal{L}}$ is logarithmic negativity, $\|\cdot\|_{1}$ stands for trace norm, and $\cdot^{T_{A}}$ refers to partial transpose with respect to party A. Since a two-mode Gaussian state is separable if and only if its partial transpose is positive ${ }^{41,42}$, it's separable if and only if its logarithmic negativity vanishes. The monotonicity of logarithmic negativity under LOCC ${ }^{43}$ (local operations with classical communication) or a PPT (positive partial transpose) operation was proved by Plenio $^{44}$, so it's a genuine entanglement monontone (or measure). The logarithmic negativity of a bipartite Gaussian state can be determined by ${ }^{37,45}$

$$
E_{\mathcal{L}}=\max \left(\log \frac{\hbar}{2 \widetilde{\nu}^{-}}, 0\right)
$$

in which $\widetilde{\nu}^{-}$is the smaller symplectic eigenvalue of the partially transposed state $\widetilde{\sigma}^{45}$ :

$$
2\left(\widetilde{\nu}^{-}\right)^{2}=\widetilde{\Delta}-\sqrt{\widetilde{\Delta}^{2}-4 \operatorname{det} \sigma},
$$

where $\Delta$ is an invariant under local symplectic transformation ${ }^{45}$

$$
\Delta \equiv \operatorname{det} A+\operatorname{det} B+2 \operatorname{det} C
$$

of the covariance matrix $\sigma=\left(\begin{array}{cc}A & C \\ C^{\mathrm{T}} & B\end{array}\right), A, B$ and $C$ being $2 \times 2$ submatrices, and $\widetilde{\Delta}$ is that of the partially transposed state, $\widetilde{\sigma}$. Note $\operatorname{det} \sigma=\operatorname{det} \widetilde{\sigma}$.

Symmetric Gaussian states. Now let's assume we have $N$ symmetric modes in a Gaussian state. The entanglement is totally determined by its covariance matrix, a $2 N \times 2 N$ matrix $^{11,12}$ :

$$
{\sigma^{\prime}}_{N}=\left(\begin{array}{cccc}
\alpha & \beta & \cdots & \beta \\
\beta & \alpha & \beta & \vdots \\
\vdots & \beta & \ddots & \beta \\
\beta & \cdots & \beta & \alpha
\end{array}\right),
$$

where $\alpha$ and $\beta$ are $2 \times 2$ real and symmetric submatrices, and the subscript $N$ is for the total number of modes. By performing $N$ appropriate local symplectic transformations on each of the $N$ modes, corresponding to $N$ local unitary transformations on the density operator, the covariance matrix (9) can always be casted into the "standard form"12,23,41,45,46:

$$
\sigma_{N}^{\prime} \rightarrow \sigma_{N}: \alpha \rightarrow \operatorname{diag}(a, a), \beta \rightarrow \operatorname{diag}(b, c),
$$

and hence irregardless of the original $\sigma^{\prime} N$, the standard form $\sigma_{N}$ and the parameters thereof, $a, b$, and $c$ will determine the block entanglement.

\section{Methods}

Orthogonal transformations for quadrature operators as a canonical transformation. Given $N$ symmetric modes at a Gaussian state whose covariance matrix is of the form (9), to scrutinize the entanglement between two blocks that have $n_{1}$ and $n_{2}$ modes respectively, the standard way is to "unitarily localize"12,23,25 these two blocks. However here we would like to treat positions and momenta separately, while still enforcing the commutation relation (canonical conjugation) upon them.

Let's consider a block with $n$ modes, $\sigma_{n}$. First, the covariances (and variances) of $\mathbf{x}=\left(x_{1}, x_{2}, \ldots, x_{n}\right)$ constitute an "x-covariance" matrix, defined as $\sigma_{i j}^{\mathbf{x}} \equiv \sigma\left(x_{i}, x_{j}\right)$, which has the same diagonal elements and the same off-diagonal ones. If we want to diagonalize it, we can utilize the fact that for an $n \times n$ matrix $M_{i j}=\varepsilon+\delta_{i j}(\lambda-\varepsilon)$, its eigenvalues are $\lambda+(n-1) \varepsilon$ and $\lambda-\varepsilon$, the latter having $(n-1)$-fold degeneracy. The eigenvector corresponding to the non-degenerate eigenvalue is $(1,1, \ldots, 1)$. That is, we can introduce these coordinates: 


$$
\mathbf{X} \equiv\left(X_{n}=\frac{1}{\sqrt{n}} \sum_{i=1}^{n} x_{i}, u_{2}, u_{3}, \ldots, u_{n}\right)=O \mathbf{x},
$$

where $O$ is an orthogonal matrix, which induces a diagonal $\mathbf{X}$-covariance matrix.

In order to maintain the commutation relation, we require the transformation for $\mathbf{p}=\left(p_{1}, p_{2}, \ldots, p_{n}\right)$ to be

$$
\mathbf{P}=\left(P_{n}=\frac{1}{\sqrt{n}} \sum_{i=1}^{n} p_{i}, \Pi_{2}, \Pi_{3}, \ldots, \Pi_{n}\right)=O \mathbf{p},
$$

where $O$ is the same orthogonal matrix as in (11). This transformation can similarly diagonalize the p-covariance matrix $\sigma_{i j}^{\mathbf{p}} \equiv \sigma\left(p_{i}, p_{j}\right)$. We can show these transformations imply

$$
\sigma\left(X_{n}, \Pi_{i}\right)=\sigma\left(u_{i}, \Pi_{j}\right)=\sigma\left(u_{i}, P_{n}\right)=0, i \neq j .
$$

To prove it, denoting $\sigma\left(x_{k}, p_{k}\right)=y$ and $\sigma\left(x_{k}, p_{l}\right)=z$ for $k \neq l$ :

$$
\begin{aligned}
\sigma\left[(\mathbf{X})_{i},(\mathbf{P})_{j}\right] & =\sum_{k, l} O_{i k} O_{j l} \sigma\left(x_{k}, p_{l}\right) \\
& =y \sum_{k} O_{i k} O_{j k}+z \sum_{k, l \neq k} O_{i k} O_{j l} \\
& =y \delta_{i j}+z \sum_{k} O_{i k} \sum_{l \neq k} O_{j l} \\
& =y \delta_{i j}+z \sum_{l} O_{j l} \sum_{k \neq l} O_{i k} .
\end{aligned}
$$

Because the rows of $O$ except for the first is orthogonal to $(1,1, \ldots, 1)$, as explained earlier, either $\sum_{k} O_{i k}=\sum_{k} 1 \cdot O_{i k}=0$ or $\sum_{l} O_{j l}=0$, or both. Hence $\sigma\left[(\mathbf{X})_{i},(\mathbf{P})_{j}\right] \neq 0$ only when $i=j$. From now on we will ignore the subscript of $u_{i}$ and $\Pi_{i}$. We can obtain ${ }^{27}$ :

$$
\begin{aligned}
\sigma\left(X_{n}, X_{n}\right) & =\sigma\left(x_{i}, x_{i}\right)+(n-1) \sigma\left(x_{i}, x_{j}\right), \\
\sigma\left(P_{n}, P_{n}\right) & =\sigma\left(p_{i}, p_{i}\right)+(n-1) \sigma\left(p_{i}, p_{j}\right), \\
\sigma(u, u) & =\sigma\left(x_{i}, x_{i}\right)-\sigma\left(x_{i}, x_{j}\right), \\
\sigma(\Pi, \Pi) & =\sigma\left(p_{i}, p_{i}\right)-\sigma\left(p_{i}, p_{j}\right), i \neq j,
\end{aligned}
$$

of which $\sigma(u, u)$ and $\sigma(\Pi, \Pi)$ doesn't depend on $n$ at all. Note $\sigma_{n}$ doesn't need to assume the standard form (10) for the results above to apply. We have effectively found a symplectic transformation that diagonalizes the covariance matrix $\sigma_{n}$.

Unitary localization of blocks. If we have two blocks with $n_{1}$ and $n_{2}$ modes, employing the same methods, we can not only diagonalize each block, but also "concentrate" all the correlations between these two blocks in $\left(X_{n_{1}}, P_{n_{1}}, X_{n_{2}}, P_{n_{2}}\right)$. Because $\sigma(u, u)$ and $\sigma(\Pi, \Pi)$ has no dependency on $n$, as shown in (15), whether a particular $\sigma(u, u)$ or $\sigma(\Pi, \Pi)$ "comes from" either block doesn't matter. The covariances with $\left(X_{n_{1}}, P_{n_{1}}, X_{n_{2}}, P_{n_{2}}\right)$ must always be zero. Therefore, we've essentially "unitarily localized" these two blocks ${ }^{12,23,25}$. Because the block entanglement is equivalent to that between two modes, having a positive partial transpose implies separability and vice versa. Hence, using negativities as the entanglement measure is certified ${ }^{12,23}$. Very recently, a novel approach to the separability problem of Gaussian states was proposed ${ }^{47}$.

To obtain the covariance matrix of the unitarily localized modes, while taking into account these two blocks are part of $N$ symmetric modes, we can make use of (15):

$$
\sigma_{N: n_{1} \mid n_{2}}=\left(\begin{array}{cc}
\alpha_{N: n_{i}} & \beta_{N: n_{1} \mid n_{2}} \\
\beta_{N: n_{1} \mid n_{2}} & \alpha_{N: n_{i}}
\end{array}\right),
$$

where $N: n_{1} \mid n_{2}$ refers to the unitary localizations of $n_{1}$ and $n_{2}$ modes that are part of $N$ modes, likewise for $N: n_{i}$, and

$$
\begin{aligned}
& \alpha_{N: n_{i}}=\operatorname{diag}[\left.\sigma\left(X_{n_{i}}, X_{n_{i}}\right), \sigma\left(P_{n_{i}}, P_{n_{i}}\right)\right] \\
&= \frac{1}{N} \operatorname{diag}\left[n_{i} \sigma\left(X_{N}, X_{N}\right)+\left(N-n_{i}\right) \sigma(u, u), n_{i} \sigma\left(P_{N}, P_{N}\right)+\left(N-n_{i}\right) \sigma(\Pi, \Pi)\right], \\
& \beta_{N: n_{1} \mid n_{2}}=\operatorname{diag}\left[\sigma\left(X_{n_{1}}, X_{n_{2}}\right), \sigma\left(P_{n_{1}}, P_{n_{2}}\right)\right] \\
&=\frac{\sqrt{n_{1} n_{2}}}{N} \operatorname{diag}\left[\sigma\left(X_{N}, X_{N}\right)-\sigma(u, u), \sigma\left(P_{N}, P_{N}\right)-\sigma(\Pi, \Pi)\right],
\end{aligned}
$$

by employing identicalness ${ }^{27}$ and by replacing $\sigma\left(x_{i}, x_{i}\right)$ and $\sigma\left(x_{i}, x_{j}\right)$ with $\sigma\left(X_{N}, X_{N}\right)$ and $\sigma(u, u)$, and replacing $\sigma\left(p_{i}, p_{i}\right)$ and $\sigma\left(p_{i}, p_{j}\right)$ with $\sigma\left(p_{N}, p_{N}\right)$ and $\sigma(\Pi, \Pi)$. (17) and (18) are diagonal by $\sigma_{N}$ taking the standard form (10). 
Parameters of a symmetric Gaussian state. The block entanglement of an $N$-mode symmetric Gaussian state can be determined by $(a, b, c)$ of (10); yet according to (17) and (18), it can be achieved also by the following four variances: $\sigma\left(X_{N}, X_{N}\right), \sigma\left(P_{N}, P_{N}\right), \sigma(u, u)$ and $\sigma(\Pi, \Pi)$, but now with one more variable. To eliminate it, we introduce the ratio:

$$
r \equiv \sigma\left(X_{N}, X_{N}\right) / \sigma(u, u)
$$

and

$$
\nu_{D} \equiv \sqrt{\sigma(u, u) \sigma(\Pi, \Pi)}, \nu_{N} \equiv \sqrt{\sigma\left(X_{N}, X_{N}\right) \sigma\left(P_{N}, P_{N}\right)} .
$$

$\nu_{D}$ and $\nu_{N}$ are actually the symplectic eigenvalues for the symmetric covariance matrix $\sigma_{N}$, with $\nu_{D}$ being degenerate, and the $N$ of $\nu_{N}$ for its $N$-dependence, c.f. (15). Hence, the formulation from (11) to (20) is just the other side of the coin of the work done by Adesso, Serafini and Illuminati ${ }^{12,23}$, who found the symplectic eigenvalues (20), but here we have one additional parameter (19) that allows us to describe a symmetric Gaussian state with these "global" parameters. Using (15), $(a, b, c)$ of $(10)$ are related to $\left(\nu_{D}, \nu_{N}, r\right)$ by, c.f. (9) and (10):

$$
\begin{aligned}
\operatorname{det} \alpha & =a^{2}=\frac{\nu_{N}^{2}(N+r-1)+\nu_{D}^{2} r(N-1)(N+r-1)}{N^{2} r}=\left(\frac{\hbar}{2 \mu_{1}}\right)^{2} \\
\operatorname{det} \beta & =b c=\frac{(1-r)\left(\nu_{D}^{2} r-\nu_{N}^{2}\right)}{N^{2} r}, \\
\operatorname{det} \sigma_{2} & =\operatorname{det}\left(\begin{array}{ll}
\alpha & \beta \\
\beta & \alpha
\end{array}\right)=\left(a^{2}-b^{2}\right)\left(a^{2}-c^{2}\right)=\frac{\nu_{D}^{2}(N+2 r-2)\left[2 \nu_{N}^{2}+\nu_{D}^{2} r(N-2)\right]}{N^{2} r}=\left(\frac{\hbar}{2}\right)^{4} \frac{1}{\mu_{2}^{2}},
\end{aligned}
$$

where $\mu_{1}$ and $\mu_{2}$ are the one- and two-mode purities ${ }^{45}$.

The advantage of applying the parameters $\left(\nu_{D}, \nu_{N}, r\right)$ over $(a, b, c)$ is that $\nu_{D} \geq \hbar / 2, \nu_{N} \geq \hbar / 2$ and $r>0$ are necessary conditions for the the state to be valid, i.e. obeying (2), therefore giving us a simpler constraint. To see that those are indeed necessary for obeying (2), note that under the aforementioned coordinate transformation, the covariance matrix is diagonalized as

$$
\operatorname{diag}\left[\left(\begin{array}{cc}
\sigma\left(X_{N}, X_{N}\right) & 0 \\
0 & \sigma\left(P_{N}, P_{N}\right)
\end{array}\right),\left(\begin{array}{cc}
\sigma(u, u) & 0 \\
0 & \sigma(\Pi, \Pi)
\end{array}\right),\left(\begin{array}{cc}
\sigma(u, u) & 0 \\
0 & \sigma(\Pi, \Pi)
\end{array}\right), \ldots,\left(\begin{array}{cc}
\sigma(u, u) & 0 \\
0 & \sigma(\Pi, \Pi)
\end{array}\right)\right] .
$$

Therefore each mode has to obey the uncertainty relation, $\sqrt{\sigma(q, q) \sigma(p, p)} \geq \hbar / 2$, leading to $\nu_{D} \geq \hbar / 2$ and $\nu_{N} \geq \hbar / 2 . r>0$ simply reflects the fact that each variance is positive, or has the same sign. Were we to define $r$ as $\sigma\left(P_{N}, P_{N}\right) / \sigma(\Pi, \Pi)$, we could obtain the same final result.

Since the ratio $\nu_{N} / \nu_{D}$ will prove important later, we replace $\nu_{N}$ with

$$
\gamma \equiv \nu_{N} / \nu_{D}
$$

which satisfies $\gamma \geq \hbar /\left(2 \nu_{D}\right)$ because $\nu_{N}=\nu_{D} \gamma \geq \hbar / 2$, and we'll use the three parameters $\left(\nu_{D}, \gamma, r\right)$ from now on, replacing $(a, b, c)$ of $(10)$.

Block Entanglement for a symmetric Gaussian state. Because the (indexes of the) blocks are interchangeable, the entanglement will depend on the sum and difference of $n_{1}$ and $n_{2}$ :

$$
n_{s} \equiv n_{1}+n_{2} \geq 2, n_{d} \equiv\left|n_{1}-n_{2}\right| \geq 0,
$$

satisfying $N \geq n_{s}>n_{d}$. It's worth pointing out that only the absolute value of $n_{1}-n_{2}$ matters, not its sign, as implied by the interchangeability. By (7) the square of the smaller symplectic eigenvalue of $\widetilde{\sigma}_{N: n_{1} \mid n_{2}}$, partial transpose of (16), is a function of $\left(\nu_{D}, \gamma, r\right)$ :

$$
f_{N: n_{s}, n_{d}} \equiv\left(\widetilde{\Delta}_{N: n_{1} \mid n_{2}}-\sqrt{\left(\widetilde{\Delta}_{N: n_{1} \mid n_{2}}\right)^{2}-4 \operatorname{det} \sigma_{N: n_{1} \mid n_{2}}}\right) / 2,
$$

where $\Delta$ is defined in (8) and $n_{s}, n_{d}$ after the colon refers to the mode numbers of the unitarily localized blocks (24). The domain of $f_{N: n_{s}, n_{d}}$ is

$$
\left\{\left(\nu_{D} \geq \hbar / 2, \gamma, r>0\right): \nu_{D} \gamma \geq \hbar / 2\right\},
$$

which is defined by the set of all valid Gaussian symmetric states. By (6) the logarithmic negativity between two blocks is

$$
E_{\mathcal{L}}^{N: n_{1} \mid n_{2}}=E_{\mathcal{L}}^{N: n_{s}, n_{d}}=\frac{1}{2} \log \max \left(\frac{\hbar^{2}}{4 f_{N: n_{s}, n_{d}}}, 1\right) ; n_{s}=n_{1}+n_{2}, n_{d}=\left|n_{1}-n_{2}\right| .
$$

Let's summarize what we have done so far: Any symmetric Gaussian state of the form (9) can be turned into the "standard form" by local unitary operations, with parameters given in (10). After orthogonal transformations on the quadrature operators and with a proper choice of parameters, the logarithmic negativity between two 
blocks (27) becomes a function with a clear domain (26). In the following subsection, we will show how this new parametrization will help us to find the suprema of negativities.

Search for the supremum/infimum. By (27) the supremum of $E_{\mathcal{L}}$, if it exists, corresponds to the infimum (greatest lower bound) of $f_{N: n_{s}, n_{d}}$, inf $f_{N: n_{s}, n_{d}}$. Hence our goal is to find this infimum. Let's start with

$$
\begin{aligned}
f_{N: n_{s}, n_{d}}\left(\nu_{D}, \gamma, r\right)= & \frac{\nu_{D}^{2}}{2 N^{2} r}\left\{\left(N n_{s}-n_{d}^{2}\right) r^{2}+\left[2 N^{2}-2 N n_{s}+n_{d}^{2}\left(1+\gamma^{2}\right)\right] r\right. \\
& +\left(N n_{s}-n_{d}^{2}\right) \gamma^{2}-\left\{\left(N n_{s}-n_{d}\right)^{2} r^{4}-2 n_{d}^{2}\left[2 N^{2}+n_{d}^{2}\left(1+\gamma^{2}\right)\right.\right. \\
& \left.-N n_{s}\left(3+\gamma^{2}\right)\right] r^{3}+\left[N^{2}\left(4 n_{d}^{2}\left(1+\gamma^{2}\right)-2 n_{s}^{2} \gamma^{2}\right)\right. \\
& \left.+n_{d}^{4}\left(\gamma^{4}+4 \gamma^{2}+1\right)-4 N n_{s} n_{d}^{2}\left(1+2 \gamma^{2}\right)\right] r^{2} \\
& -2 n_{d}^{2}\left[2 N^{2}+n_{d}^{2}\left(1+\gamma^{2}\right)-N n_{s}\left(3+\gamma^{2}\right)\right] \gamma^{2} r+\left(N n_{s}-n_{d}^{2}\right)^{2} \\
& \left.\left.\gamma^{4}\right\}^{1 / 2}\right\},
\end{aligned}
$$

continuous everywhere on the domain (26). With three variables, first let's vary $r$ only while keeping $\nu_{D}$ and $\gamma$ fixed, by defining

$$
F_{\nu_{D}, \gamma}^{N: n_{s}, n_{d}}(r) \equiv f_{N: n_{s}, n_{d}}\left(\nu_{D}, \gamma, r\right)
$$

as a function of $r$ only. $F$ is of this form (henceforth we'll ignore the superscripts and subscripts of $f$ and $F$ ):

$$
F(r)=\frac{\nu_{D}^{2}}{N^{2}} \frac{p(r)-\sqrt{h(r)}}{r},
$$

where $p$ and $h$ are polynomials of second and forth orders respectively, whose coefficients are made up of $\left(\gamma, N, n_{s}\right.$, $n_{d}$ ), without $\nu_{D}$. Because $F(r)$ is a differentiable function of $r \in(0, \infty)$, the global minimum or infimum lies on the boundary or where the derivative vanishes. Let us first examine the behavior of $F(r)$ at the boundary.

Boundary. Using L'Hôpital's rule, the limits of $F$ as $r$ approaches the boundary 0 and $\infty$ are

$$
\lim _{r \rightarrow 0} F(r)=\lim _{r \rightarrow \infty} F(r)=\nu_{D}^{2} \frac{N n_{s}-n_{s}^{2}}{N n_{s}-n_{d}^{2}},
$$

independent of $\gamma$, or $\nu_{N}$. Note it's nonnegative and smaller than $\nu_{D}^{2}$ because $N \geq n_{s}>n_{d}$.

Critical point. Next, we shall investigate where $F^{\prime}(r)=0$. By applying the lemma that can be found in the supplementary material, the critical point is at $r=\gamma$. In addition, $F(0)=F(\infty)$ (which will denote $\lim _{r \rightarrow 0} F(r)$ and $\lim _{r \rightarrow \infty} F(r)$ hereafter), differentiability of $F(r)$, and the existence of only one critical point imply that $F(\gamma)$ is either a global maximum or minimum, and that $F(0)=F(\infty)$ is either the supremum or infimum; that is, the smaller one of $F(0)=F(\infty)$ and $F(\gamma)$ is the infimum of $F$.

Value at the critical point. However, instead of directly comparing $F(0)=F(\infty)$ and $F(\gamma)$, let's see how small $F(\gamma)$ can be, because if $F(\gamma) \geq \hbar^{2} / 4$, then the entanglement measure would be zero by (27); namely, this point is of no interest to us at all! By (25),

$$
\begin{aligned}
F(\gamma)= & \frac{\nu_{D}^{2}}{2 N^{2}}\left[2 N^{2}+2 N n_{s}(\gamma-1)+n_{d}^{2}(\gamma-1)^{2}\right. \\
& \left.-n_{d}|\gamma-1| \sqrt{4 N^{2}+4 N n_{s}(\gamma-1)+n_{d}^{2}(\gamma-1)^{2}}\right] .
\end{aligned}
$$

Let's define

$$
g(\gamma) \equiv F(r=\gamma)
$$

as a continuous function of $\gamma$, and

$$
q \equiv \hbar /\left(2 \nu_{D}\right) \in(0,1] .
$$

Because $\nu_{N}=\nu_{D} \gamma \geq \hbar / 2$ by (23), $\gamma \geq q$. We consider two cases separately for $g(\gamma)$, i.e. $\gamma>1$ and $1 \geq \gamma \geq q$ :

1. $\gamma>1$ : Defining $x \equiv \gamma-1>0$, we try to solve $g(\gamma=x+1)<\hbar^{2} / 4$ with the constraint $q \leq 1$. This leads to a quadratic inequality $c_{2} x^{2}+c_{1} x+c_{0}<0$, where $c_{2}>0, c_{1} \geq 0$ and $c_{0} \geq 0$. Hence, it has no nonnegative solutions. More details can be found in the supplementary material.

2. $1 \geq \gamma \geq q$ : Defining $x \equiv 1-\gamma \in[0,1-q]$, the solution of $g(\gamma=1-x)<\hbar^{2} / 4$ is the interval 


$$
\left(\frac{N\left(1-q^{2}\right)}{n_{s}+n_{d} q}, \frac{N\left(1-q^{2}\right)}{n_{s}-n_{d} q}\right) .
$$

Since the greatest lower bound of the interval above

$$
\frac{N\left(1-q^{2}\right)}{n_{s}+n_{d} q}=\frac{N(1+q)}{n_{s}+n_{d} q}(1-q) \geq 1-q
$$

by $N \geq n_{s}>n_{d} \geq 0$ and $q>0$, no solution exists for $x \in[0,1-q]$.

Therefore, $g(\gamma)=F(\gamma) \geq \hbar^{2} / 4$ on the domain $\gamma \in[q, \infty)$, so when $\inf F$ is smaller than $\hbar^{2} / 4$, inf $F$ is definitely $F(0)=F(\infty)$.

Data availability. No data was generated or analyzed for this study.

\section{Results}

Thanks to the continuity of $f,(28), \forall \varepsilon>0$ as $\left(\nu_{D}, \gamma\right) \rightarrow\left(\nu_{D}^{\prime}, \gamma^{\prime}\right)$

$$
f\left(\nu_{D}, \gamma, \varepsilon\right) \rightarrow f\left(\nu_{D}^{\prime}, \gamma^{\prime}, \varepsilon\right),
$$

so as $\left(\nu_{D}, \gamma, r\right) \rightarrow\left(\nu^{\prime}{ }_{D}, \gamma^{\prime}, 0\right)$

$$
f\left(\nu_{D}, \gamma, r\right) \rightarrow f\left(\nu_{D}^{\prime}, \gamma^{\prime}, r\right) \rightarrow \lim _{r \rightarrow 0} F_{\nu^{\prime}, \gamma^{\prime}}(r),
$$

i.e. the limit as $r \rightarrow 0$ is identical for $f$ and $F$, same if $r \rightarrow \infty$. Hence, when $\inf F<\hbar^{2} / 4, F(0)=F(\infty)$ is the infimum of $f$ at a fixed $\nu_{D}$, for $F(0)=F(\infty)$ is independent of $\gamma$, or $\nu_{N}$. From (27) and (31), we have:

Proposition 1: For $N$ modes in a symmetric Gaussian state at a given $\nu_{D}$, the degenerate symplectic eigenvalue (20), the supremum of logarithmic negativity between two blocks containing $n_{1}$ and $n_{2}$ modes is

$$
\sup E_{\mathcal{L}: \nu_{D}}^{N: n_{1} \mid n_{2}}=E_{\mathcal{L}: \nu_{D}}^{N: n_{s} n_{d}}=\max \left[\frac{1}{2} \log \frac{\hbar^{2}}{4 \nu_{D}^{2}}\left(1+\frac{n_{s}^{2}-n_{d}^{2}}{n_{s}\left(N-n_{s}\right)}\right), 0\right] ; n_{s}=n_{1}+n_{2}, n_{d}=\left|n_{1}-n_{2}\right| .
$$

Likewise, because the infimum of $f\left(\nu_{D}, \gamma, r\right)$ over all $\left(\nu_{D}, \gamma, r\right)$ is the infimum of $f\left(\nu_{D}, \gamma, 0\right)=F_{\nu_{D}, \gamma}(0)$ over all $\left(\nu_{D}, \gamma\right)$ and because $\nu_{D} \geq \hbar / 2$, we obtain:

Proposition 2: Using the same notations as in proposition 1, for all symmetric Gaussian states:

$$
\sup E_{\mathcal{L}}^{N: n_{s}, n_{d}}=\frac{1}{2} \log \left(1+\frac{n_{s}^{2}-n_{d}^{2}}{n_{s}\left(N-n_{s}\right)}\right) .
$$

Note (40) is always positive, while (39) is nonnegative. Similar results apply to negativity $E_{\mathcal{N}}{ }^{37}$ by the relation $E_{\mathcal{N}}=\left(t^{E_{\mathcal{L}}}-1\right) / 2$, assuming the base is $t$. Namely,

Proposition 3: Following the notations like proposition 1, for all symmetric Gaussian states the negativity between two blocks has a supremum:

$$
\sup E_{\mathcal{N}}^{N: n_{s}, n_{d}}=\frac{1}{2}\left[\left(1+\frac{n_{s}^{2}-n_{d}^{2}}{n_{s}\left(N-n_{s}\right)}\right)^{1 / 2}-1\right] .
$$

At a given $\nu_{D}$ :

$$
\sup E_{\mathcal{N}: \nu_{D}}^{N: n_{s}, n_{d}}=\max \left\{\frac{1}{2}\left[\frac{\hbar}{2 \nu_{D}}\left(1+\frac{n_{s}^{2}-n_{d}^{2}}{n_{s}\left(N-n_{s}\right)}\right)^{1 / 2}-1\right], 0\right\}
$$

Furthermore, by intermediate value theorem ${ }^{48,49}$, with two blocks at given $\left(N, n_{s}, n_{d}\right)$ and perhaps $\nu_{D}$, for all elements in the following interval:

$$
[0, \sup E)
$$

where $\sup E$ is (39), (40), (41) or (42) depending on whether $\nu_{D}$ is fixed and which measure is adopted, we can always find a corresponding symmetric Gaussian state, unless $\sup E=0$ (which can only happen with fixed $\nu_{D}$, (39) and (42)), in which case the entanglement measure can only be zero. In other words, the mapping from a symmetric Gaussian state to the interval (43) is surjective (but not injective). Note these suprema aren't maxima, so the interval (43) is not closed. 


\section{Discussions}

Boundedness and block sizes. Suppose we have two blocks, with $n_{1}$ and $n_{2}$ modes, from $N$ symmetric modes. As $N$ increases, the supremum of bipartite entanglement between them decreases, unless $n_{1}+n_{2}=N$. What is the reason behind? By increasing the total number of interchangeable modes, we actually impose a stronger constraint on individual modes, i.e. $(a, b, c)$ of (10). This should be clearer with the help of (15): In terms of the parameters $\left(\nu_{D}, \nu_{N}, r\right)$ as defined by (19) and (20), which is interchangeable with $(a, b, c)$, we can find as $r \rightarrow 0$ or $r \rightarrow \infty$

$$
\sigma\left(X_{N^{\prime}}, X_{N^{\prime}}\right) \sigma\left(P_{N^{\prime}}, P_{N^{\prime}}\right) \rightarrow\left\{\begin{array}{cl}
-\infty & \text { if } N^{\prime}>N \\
\nu_{N} & \text { if } N^{\prime}=N \\
\infty & \text { if } N^{\prime}<N
\end{array}\right.
$$

This implies that at both limits the symmetric state remains legitimate with $N$ total modes or less, but becomes invalid if there are $N^{\prime}>N$ symmetric modes in total, violating (2); hence we're left with "less" choices (still infinite) of $(a, b, c)$ as $N$ grows. Please heed that with the same "local state", or $(a, b, c)$, the entanglement between two blocks of given sizes should be the same, irregardless of how large $N$ is: What $N$ does is restricting the choice of "local states", which in turn restricts entanglement. A valid local state for $N=N_{1}$ may be invalid for $N=N_{2}>N_{1}$.

In the supplementary material, we prove that for all symmetric Gaussian states, at fixed $n_{s}$ and $N$ a larger $n_{d}$ decreases the negativities of block entanglement, which was observed by Serafini et al. ${ }^{12}$ The suprema (39) (42) comply with this phenomenon as well: As the blocks become more equal, they become more entangled. This suggests an optimal strategy of "gathering" entanglement ${ }^{12}$.

At the same state, the block entanglement between subsystems should be smaller than or equal to that between the systems. This is reflected by the fact that a larger $n_{s}$ implies larger suprema, which can be checked by partially differentiating them. Furthermore, we can replace $n_{d}$ and $n_{s}$ with $n_{1}$ and $n_{2}$, and find that these suprema do increase by adding more modes to either block.

Monogamy of entanglement and multipartite entanglement. Monogamy of entanglement ${ }^{50,51}$, describing the phenomenon that entanglement between multiple parties can't be shared freely, can be embodied by an inequality first introduced by Coffman, Kundu, and Wooters $(\mathrm{CKW})^{50,52}$ :

$$
E^{p_{1} \mid\left(p_{2}, \ldots, p_{N}\right)} \geq \sum_{i=1}^{N} E^{p_{1} \mid p_{N}}
$$

where $p_{i}$ denotes the $i$-th party, and $E^{p_{i}} \mid p_{j}$ is the bipartite entanglement between party $i$ and party $j$. In the tripartite case, the difference between the left and right hand sides of the inequality above is the residual entanglement, characterizing the tripartite entanglement ${ }^{14}$. However, not all genuine entanglement measures obey this inequality; instead, a class of entanglement measures has been chosen to satisfy this condition, such as tangle ${ }^{50}$ and cotangle $^{14,53}$. A stronger form of entanglement monogamy was also proposed ${ }^{25,54}$ :

$$
E^{p_{1} \mid\left(p_{2}, \ldots, p_{N}\right)}=\sum_{j=2}^{N} E^{p_{1} \mid p_{j}}+\sum_{k>j=2}^{N} E^{p_{1}\left|p_{j}\right| p_{k}}+\cdots+E \underline{p_{1}}\left|p_{2}\right| \cdots \mid p_{N},
$$

where the second and latter terms are all multipartite entanglements with more than two parties. The genuine residual $N$-partite entanglement $E^{p_{1}}\left|p_{2}\right| \ldots \mid p_{N}$ can be found by minimizing $E \underline{p}_{1}\left|p_{2}\right| \ldots \mid p_{N}$, choosing the appropriate probing party $p_{i}^{25,54}$. In principle an $N$-partite entanglement can be attained by iteration, with the information of bipartite entanglements ${ }^{25,54}$.

Even though negativity and logarithmic negativity, the focus of our present paper, in general don't obey CKW inequality ${ }^{14}$, our results show some interesting aspects regarding Gaussian multipartite entanglement: The bipartite block entanglement, with respect to negativities, becomes unbounded when $n_{s}=N$, bounded otherwise. If we have $m$ blocks from $N$ symmetric modes, with $n_{1}+\cdots+n_{m}<N$, then since all bipartite entanglement between them is bounded, (46) implies that the $m$-partite entanglement among these $m$ blocks is also bounded. On the other hand, if $n_{1}+\cdots+n_{m}=N$, because the bipartite entanglement between one and the remaining parties is unbounded, we expect the multipartite entanglement involving all of them is unbounded as well. In short, if the blocks fill all the symmetric modes, the multipartite entanglement is expected to be unbounded; bounded if not.

A few more words can be also said on the boundedness: Because when $n_{1}+n_{2}=N$ the entanglement is unbounded, that the maximal entanglement between blocks of given sizes decreases with increasing $N$ isn't due to a limited amount of entanglement distributed to more parties (by (45), the monogamy of entanglement), leading to every party gaining less: There's (potentially) infinite entanglement to begin with.

Rigorously speaking, this problem should be treated with a suitable measure that obeys (45), like cotangle. However, this is beyond our current scope, and we hope this primitive discussion can inspire future works.

Available entanglement. Because logarithm is monotonic, how sup E behaves depends on (c.f. (39) (42))

$$
K\left(N, n_{s}, n_{d}\right) \equiv \frac{n_{s}^{2}-n_{d}^{2}}{n_{s}\left(N-n_{s}\right)} .
$$

For $n_{s}<N$, the greatest supremum can be achieved when $n_{s}=N-1$, with the corresponding $K$ being 


$$
K=\left\{\begin{array}{ll}
N-1 & \text { for odd } N \text { and } n_{d}=0 \\
N-1-1 /(N-1) & \text { for even } N \text { and } n_{d}=1
\end{array},\right.
$$

which clearly becomes larger as $N$ increases. Therefore, excluding the case $n_{s}=N$, as the total number of symmetric modes $N$ increases, we can actually have more entanglement at out disposal.

By (39) and (42) at a given $\nu_{D}$ no entanglement can exist between any two blocks if

$$
K\left(N, n_{s}, n_{d}\right)+1 \leq \frac{4 \nu_{D}^{2}}{\hbar^{2}},
$$

so by (48) when

$$
\frac{4 \nu_{D}^{2}}{\hbar^{2}} \geq\left\{\begin{array}{ll}
N & \text { for odd } N \\
N-1 /(N-1) & \text { for even } N
\end{array},\right.
$$

two blocks with $n_{s}<N$ can only be separable; in other words for symmetric modes with $\nu_{D}$ satisfying the condition above any two blocks are separable unless $n_{s}=N$.

Purities. If we can gain information of $\nu_{D}$, then it's possible to lower the supremum, i.e. using (39) instead of (40). A symmetric Gaussian pure state has $\nu_{D}=\nu_{N}=\hbar / 2$, and for bisymmetric ${ }^{12}$ or multisymmetric Gaussian states ${ }^{25}$ composed of several clusters of symmetric modes $\nu_{D}=\hbar / 2$, so for those states the upper bound cannot be lowered. Because the global purity $\mu$ of symmetric Gaussian modes is ${ }^{23,45}$

$$
\mu=\frac{1}{\nu_{N} \nu_{D}^{N-1}}\left(\frac{\hbar}{2}\right)^{N}=\frac{1}{\gamma}\left(\frac{\hbar}{2 \nu_{D}}\right)^{N}
$$

which fixes the parameter $\gamma$. However, since the the suprema lie on the boundary $r \rightarrow 0$ and $r \rightarrow \infty, \gamma$ doesn't really play a role in determining the values of the suprema. Hence if the global purity is known, which even though can "shrink" the domain, the suprema stay the same.

As a comparison, Adesso et al. ${ }^{11}$ found that the entanglement between two modes of a Gaussian state is bounded from below and above if the global (two-mode) and marginal (one-mode) purities are known. However, were we to ascertain the bounds imposed by purities of multiple modes (e.g. global purity) and of one mode for symmetric Gaussian states, it either would constitute a complicated constraint of the domain for the current parameters in use, i.e. $\left(\nu_{D}, \gamma, r\right)$ or $(a, b, c)$ of $(10)$, or while reduces it to a one-variable problem, would require a suitable parameter and technique to find the bounds, and hence not the subject of our work at this stage.

Two modes and approximation. Next we consider the entanglement between two modes as a special case. When $n_{1}=n_{2}=1$ (40) becomes

$$
\sup E_{\mathcal{L}}^{N: 1 \mid 1}=\frac{1}{2} \log \left(1+\frac{2}{N-2}\right)
$$

as obtained in our previous work ${ }^{27}$, which was proved for a bisymmetric ${ }^{12}$ Gaussian pure state under the assumption that the coefficients of the wave function are real, so in the present work we provide a complete proof and a more general result. Taking the base to be $e$, it has an approximation $1 / N$ as $N \gg 1$.

More generally, when $N \gg n_{s}$ (and thus $N \gg n_{d}$ ) (40) approximates

$$
\sup E_{\mathcal{L}}^{N: n_{s}, n_{d}} \simeq \frac{n_{s}^{2}-n_{d}^{2}}{2 n_{s} N} \simeq \frac{n_{s}}{2 N},
$$

where the second approximation can be made if $n_{d} \ll n_{s}$ or $n_{d}=0$. By the same token, we acquire $\sup E_{\mathcal{N}}^{N: n_{1} \mid n_{2}} \simeq\left(n_{s}^{2}-n_{d}^{2}\right) /\left(4 n_{s} N\right)$, and $\sup E_{\mathcal{N}}^{N: n_{1} \mid n_{2}} \simeq n_{s} /(4 N)$ when $n_{d} \ll n_{s}$. Here scaling with respect to $N$ and $n_{s}$ is demonstrated in an explicit manner: proportional to $n_{s}$ and inversely proportional to $N$ when $N \gg n_{s}$.

\section{Conclusion}

We have shown the least upper bounds of block entanglement for Gaussian state with $N$-symmetric modes, in terms of logarithmic negativity and negativity, (40) and (41); if $\nu_{D}$ of (20), the degenerate symplectic eigenvalue can be known, (39) and (42) for tighter bounds. Such upper bounds originates from the symmetry of the state, and the basic requirement that the state obey the uncertainty relation, satisfying (2). These two conditions together impose a constraint on the modes, which becomes more stringent for each individual mode as the total number of modes $N$ increases; that is, with "local" parameters like $(a, b, c)$ of $(10)$ to describe a symmetric Gaussian state, the domain shrinks as a result of increasing $N$.

Despite the dwindling domain of local parameters, the possibility for the blocks to swell even more, i.e. larger $n_{s}=n_{1}+n_{2}$ can not only compensate for that, but the achievable maximal block entanglement, or unitarily localizable entanglement can increases as a result of increasing total modes, (48), at the expense of entanglement between blocks of fixed sizes, say $n_{1}=n_{2}=1$, c.f. some previous works ${ }^{12,23}$. Yet at whichever $N$, for two blocks with $n_{1}+n_{2}=N$, there exists no upper bound for their block entanglement. In other words, the unitarily localizable entanglement will be wasted if it's not gathered across all the symmetric modes, but if the localization can be done 
in full, then the total number of symmetric modes makes little difference, in terms of the amount of available resource that is entanglement. Moreover, by the "strong" monogamy of entanglement, the same can be anticipated for $m$-partite entanglement, i.e. whether it's bounded depends on if $n_{1}+n_{2}+\cdots+n_{m}=N$.

Gaussian states, as a subset of states of an infinite-dimensional space, have an unbounded entanglement (with respect to measures like negativities) if there's no constraint put on them. In this regard, symmetry under mode swapping is a very strong condition, which renders the entanglement bounded. Ascertaining the exact bound was then made possible by choosing global parameters with a clear boundary, (26). The method employed in this work may be helpful in other related issues, where (multi)symmetric Gaussian states are the subject.

\section{References}

1. Nielsen, M. A. \& Chuang, I. L. Quantum Computation and Quantum Information: 10th Anniversary Edition. (Cambridge University Press, Cambridge, 2011).

2. Bell, J. On the einstein podolsky rosen paradox. Physics 1, 195-200, https://cds.cern.ch/record/111654/files/vollp195-200_001.pdf (1964).

3. Brunner, N., Cavalcanti, D., Pironio, S., Scarani, V. \& Wehner, S. Bell nonlocality. Rev. Mod. Phys. 86, 419-478, https://doi. org/10.1103/RevModPhys.86.419 (2014).

4. Mandel1, O. et al. Quantum thermalization through entanglement in an isolated many-body system. Nature 425, 937-940, https:// www.nature.com/nature/journal/v425/n6961/full/nature02008.html (2013).

5. Horodecki, R., Horodecki, P., Horodecki, M. \& Horodecki, K. Quantum entanglement. Rev. Mod. Phys. 81, 865-942, https://doi. org/10.1103/RevModPhys.81.865 (2009).

6. Louchet-Chauvet, A. et al. Entanglement-assisted atomic clock beyond the projection noise limit. New J. Phys. 12, 065032, http:// stacks.iop.org/1367-2630/12/i=6/a=065032 (2010).

7. Pan, J.-W. et al. Multiphoton entanglement and interferometry. Rev. Mod. Phys. 84, 777-838, https://doi.org/10.1103/ RevModPhys.84.777 (2012).

8. Cramer, M. et al. Spatial entanglement of bosons in optical lattices. Nat. Comms 4, 2161, https://www.nature.com/articles/ ncomms3161 (2013).

9. Aolita, L. Open-system dynamics of entanglement:a key issues review. Rep. Prog. Phys. 78, 042001, https://doi.org/10.1088/00344885/78/4/042001 (2015).

10. Kaufman, A. M. et al. Quantum thermalization through entanglement in an isolated many-body system. Science 353, 794-800, http://science.sciencemag.org/content/353/6301/794 (2016).

11. Adesso, G., Serafini, A. \& Illuminati, F. Determination of continuous variable entanglement by purity measurements. Phys. Rev. Lett. 92, 087901, https://doi.org/10.1103/PhysRevLett.92.087901 (2004).

12. Serafini, A., Adesso, G. \& Illuminati, F. Unitarily localizable entanglement of gaussian states. Phys. Rev. A 71, 032349, https://doi. org/10.1103/PhysRevA.71.032349 (2005)

13. Braunstein, S. L. \& van Loock, P. Quantum information with continuous variables. Rev. Mod. Phys. 77, 513-577, https://doi. org/10.1103/RevModPhys.77.513 (2005).

14. Adesso, G. \& Illuminati, F. Continuous variable tangle, monogamy inequality, and entanglement sharing in gaussian states of continuous variable systems. New J. Phys. 8, 15, http://stacks.iop.org/1367-2630/8/i=1/a=015 (2006).

15. Serafini, A. Multimode uncertainty relations and separability of continuous variable states. Phys. Rev. Lett. 96, 110402, https://doi. org/10.1103/PhysRevLett.96.110402 (2006).

16. Fiurášek, J. \& Mišta, L. Gaussian localizable entanglement. Phys. Rev. A 75, 060302, https://doi.org/10.1103/PhysRevA.75.060302 (2007).

17. Adesso, G. \& Illuminati, F. Entanglement in continuous-variable systems: recent advances and current perspectives. J. Phys. A: Math. Theor. 40, 7821, http://stacks.iop.org/JPhysA/40/7821 (2007).

18. Giorda, P. \& Paris, M. G. A. Gaussian quantum discord. Phys. Rev. Lett. 105, 020503, https://doi.org/10.1103/PhysRevLett.105.020503 (2010).

19. Adesso, G. \& Datta, A. Quantum versus classical correlations in gaussian states. Phys. Rev. Lett. 105, 030501, https://doi.org/10.1103/ PhysRevLett.105.030501 (2010)

20. Serafini, A. \& Mancini, S. Determination of maximal gaussian entanglement achievable by feedback-controlled dynamics. Phys. Rev. Lett. 104, 220501, https://doi.org/10.1103/PhysRevLett.104.220501 (2010).

21. Weedbrook, C. et al. Gaussian quantum information. Rev. Mod. Phys. 84, 621-669, https://doi.org/10.1103/RevModPhys.84.621 (2012).

22. Adesso, G., Ragy, S. \& Lee, A. R. Continuous variable quantum information: Gaussian states and beyond. Open Syst. Inf. Dyn. 21, 1440001, https://arxiv.org/abs/1401.4679 (2014).

23. Adesso, G., Serafini, A. \& Illuminati, F. Quantification and scaling of multipartite entanglement in continuous variable systems. Phys. Rev. Lett. 93, 220504, https://doi.org/10.1103/PhysRevLett.93.220504 (2004).

24. Adesso, G., Serafini, A. \& Illuminati, F. Entanglement, purity, and information entropies in continuous variable systems. Open Syst. Inf. Dyn. 12, 189, https://arxiv.org/abs/quant-ph/0506049 (2005).

25. Adesso, G. \& Illuminati, F. Genuine multipartite entanglement of symmetric gaussian states: Strong monogamy, unitary localization, scaling behavior, and molecular sharing structure. Phys. Rev. A 78, 042310, https://doi.org/10.1103/PhysRevA.78.042310 (2008).

26. Xu, B., Tufarelli, T. \& Adesso, G. Genuine multipartite nonlocality of permutationally invariant gaussian states. Phys. Rev. A 95, 012124, https://doi.org/10.1103/PhysRevA.95.012124 (2017).

27. Kao, J.-Y. \& Chou, C.-H. Quantum entanglement in coupled harmonic oscillator systems: from micro to macro. New J. Phys. 18, 073001, http://stacks.iop.org/1367-2630/18/i=7/a=073001 (2016).

28. Bennett, C. H., DiVincenzo, D. P., Smolin, J. A. \& Wootters, W. K. Mixed-state entanglement and quantum error correction. Phys. Rev. A 54, 3824-3851, https://doi.org/10.1103/PhysRevA.54.3824 (1996).

29. Rigolin, G. \& Escobar, C. O. Lower bounds on the entanglement of formation for general gaussian states. Phys. Rev. A 69, 012307, https://doi.org/10.1103/PhysRevA.69.012307 (2004).

30. Nicacio, F. \& de Oliveira, M. C. Tight bounds for the entanglement of formation of gaussian states. Phys. Rev. A 89, 012336, https:// doi.org/10.1103/PhysRevA.89.012336 (2014).

31. Wei, T.-C. \& Goldbart, P. M. Geometric measure of entanglement and applications to bipartite and multipartite quantum states. Phys. Rev. A 68, 042307, https://doi.org/10.1103/PhysRevA.68.042307 (2003).

32. Barnum, H. \& Linden, N. Monotones and invariants for multi-particle quantum states. J. Phys. A 34, 6787, http://stacks.iop. org/0305-4470/34/i=35/a=305 (2001).

33. Shimony, A. Degree of entanglement. Annals of the New York Academy of Sciences 755, 675-679, https://doi.org/10.1111/j.1749-6632.1995. tb39008.x (1995).

34. Martin, J., Giraud, O., Braun, P. A., Braun, D. \& Bastin, T. Multiqubit symmetric states with high geometric entanglement. Phys. Rev. A 81, 062347, https://doi.org/10.1103/PhysRevA.81.062347 (2010). 
35. Aulbach, M., Markham, D. \& Murao, M. The maximally entangled symmetric state in terms of the geometric measure. New J. Phys. 12, 073025, http://stacks.iop.org/1367-2630/12/i=7/a =073025 (2010).

36. Aulbach, M. Classification of entanglement in symmetric states. Int. J. Quantum Inform. 10 (2012).

37. Vidal, G. \& Werner, R. F. Computable measure of entanglement. Phys. Rev. A 65, 032314, https://doi.org/10.1103/ PhysRevA.65.032314 (2002).

38. Simon, R., Mukunda, N. \& Dutta, B. Quantum-noise matrix for multimode systems: U(n) invariance, squeezing, and normal forms. Phys. Rev. A 49, 1567-1583, https://doi.org/10.1103/PhysRevA.49.1567 (1994).

39. Peres, A. Separability criterion for density matrices. Phys. Rev. Lett. 77, 1413-1415, https://doi.org/10.1103/PhysRevLett.77.1413 (1996).

40. Horodecki, P. Separability criterion and inseparable mixed states with positive partial transposition. Phys. Lett. A 232, 333-339, http://www.sciencedirect.com/science/article/pii/S0375960197004167 (1997).

41. Simon, R. Peres-horodecki separability criterion for continuous variable systems. Phys. Rev. Lett. 84, 2726-2729, https://doi. org/10.1103/PhysRevLett.84.2726 (2000).

42. Werner, R. F. \& Wolf, M. M. Bound entangled gaussian states. Phys. Rev. Lett. 86, 3658-3661, https://doi.org/10.1103/PhysRevLett.86.3658 (2001).

43. Chitambar, E., Leung, D., Mancinska, L., Ozols, M. \& Winter, A. Everything you always wanted to know about locc (but were afraid to ask). Commun. Math. Phys. 328, 303-326, https://link.springer.com/article/10.1007 (2014).

44. Plenio, M. B. Logarithmic negativity: A full entanglement monotone that is not convex. Phys. Rev. Lett. 95, 090503, https://doi. org/10.1103/PhysRevLett.95.090503 (2005).

45. Serafini, A., Illuminati, F. \& Siena, S. D. Symplectic invariants, entropic measures and correlations of gaussian states. J. Phys. B: At. Mol. Opt. Phys. 37, L21, http://stacks.iop.org/0953-4075/37/i=2/a=L02 (2004).

46. Duan, L.-M., Giedke, G., Cirac, J. I. \& Zoller, P. Inseparability criterion for continuous variable systems. Phys. Rev. Lett. 84, 2722-2725, https://doi.org/10.1103/PhysRevLett.84.2722 (2000).

47. Lami, L., Serafini, A. \& Adesso, G. Gaussian entanglement revisited. New J. Phys. 20, 023030, http://stacks.iop.org/1367-2630/20/ $\mathrm{i}=2 / \mathrm{a}=023030$ (2018).

48. Duistermaat, J. J. \& Kolk, J. A. C. Multidimensional Real Analysis I: Differentiation. (Cambridge University Press, Cambridge, 2004).

49. Kantorovitz, S. Several Real Variables (Springer, 2016).

50. Coffman, V., Kundu, J. \& Wootters, W. K. Distributed entanglement. Phys. Rev. A 61, 052306, https://doi.org/10.1103/ PhysRevA.61.052306 (2000).

51. Terhal, B. M. Is entanglement monogamous? IBM J. Res. Dev. 48, 71-78, https://arxiv.org/abs/quant-ph/0307120 (2004).

52. Adesso, G. \& Illuminati, F. Entanglement sharing: from qubits to gaussian states. Int. J. Quant. Inf 4, 383-393, https://arxiv.org/abs/ quant-ph/0506213 (2006).

53. Hiroshima, T., Adesso, G. \& Illuminati, F. Monogamy inequality for distributed gaussian entanglement. Phys. Rev. Lett. 98, 050503, https://doi.org/10.1103/PhysRevLett.98.050503 (2007)

54. Adesso, G. \& Illuminati, F. Strong monogamy of bipartite and genuine multipartite entanglement: The gaussian case. Phys. Rev. Lett. 99, 150501, https://doi.org/10.1103/PhysRevLett.99.150501 (2007).

\section{Acknowledgements}

This work is partly supported by Ministry of Science and Technology of Taiwan under the grant number MOST 105-2112-M-006-011. This reserach is also supported in part by Headquarters of University Advancement at the National Cheng Kung University, which is sponsored by the Ministry of Education, Taiwan.

\section{Author Contributions}

J.-Y. Kao performed the derivation and composed the first draft of this article. C.-H. Chou supervised this project. Both authors contributed to the writing and editing of the manuscript.

\section{Additional Information}

Supplementary information accompanies this paper at https://doi.org/10.1038/s41598-018-25781-2.

Competing Interests: The authors declare no competing interests.

Publisher's note: Springer Nature remains neutral with regard to jurisdictional claims in published maps and institutional affiliations.

(c) (i) Open Access This article is licensed under a Creative Commons Attribution 4.0 International

License, which permits use, sharing, adaptation, distribution and reproduction in any medium or format, as long as you give appropriate credit to the original author(s) and the source, provide a link to the Creative Commons license, and indicate if changes were made. The images or other third party material in this article are included in the article's Creative Commons license, unless indicated otherwise in a credit line to the material. If material is not included in the article's Creative Commons license and your intended use is not permitted by statutory regulation or exceeds the permitted use, you will need to obtain permission directly from the copyright holder. To view a copy of this license, visit http://creativecommons.org/licenses/by/4.0/.

(C) The Author(s) 2018 